\title{
Error and modification in thermal barrier coatings measurement using impedance spectroscopy
}

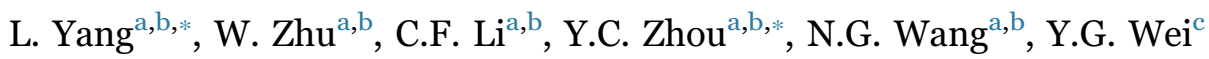 \\ a Key Laboratory of Key Film Materials \& Application for Equipment (Hunan province), School of Materials Science and Engineering, Xiangtan University, \\ Xiangtan, Hunan 411105, China \\ ${ }^{\mathrm{b}}$ Key Laboratory of Low Dimensional Materials and Application Technology of Ministry of Education, School of Materials Science and Engineering, \\ Xiangtan University, Xiangtan, Hunan 411105, China \\ ${ }^{\mathrm{c}}$ LNM, Institute of Mechanics, Chinese Academy of Sciences, Beijing 100190, China
}

\section{A R T I C L E I N F O}

\section{Keywords:}

Impedance spectroscopy

Asymmetric electrode

Divergence of electric field

Thermal barrier coating

Measurement error

\begin{abstract}
A B S T R A C T
In impedance spectroscopy testing of metal/ceramic multi-layers, such as thermal barrier coatings (TBCs), in order to avoid electric leakage, asymmetric electrode is widely adopted. However, how is electric field distribution in asymmetric electrode system, what error does it bring to measurement results, and how to modify these errors etc., are still not understood. In this study, electric field divergence in TBCs measurement induced by asymmetric electrode is investigated through solving a three-dimensional electric field equations numerically. The results show that the asymmetric electrode inevitably triggers divergence of electric field line, and as frequency decreases the divergence increases considerably. When Pt electrode diameter is chosen as $1 \mathrm{~mm}$ in ceramic coating, the errors in thickness measurement for both YSZ and TGO are 26.20\% and 89.3\%, respectively. The errors rise as both YSZ and TGO thicknesses increase, while the errors decrease as Pt electrode size increases. The error in thickness measurement for TGO layer is much larger than that for YSZ. Through present research, a scheme to eliminate the effect of electric field divergence on measurement error is proposed.
\end{abstract}

\section{Introduction}

Thermal barrier coatings (TBCs), as one of the most important key techniques for thermal protection of aero engines, have been developed as the most feasible solution to improve the service temperature of aero engines, which can improve the efficiency of power generation gas turbines and prolong the lifetime of turbine blades [1,2]. Due to their low thermal conductivity, a temperature drop can be attained up to $200{ }^{\circ} \mathrm{C}$ through thermal isolation in TBCs as well as an inner cooling system $[1,2]$. During service of gas turbines, TBCs is subjected to high temperature exposure, sintering and corrosion, which can lead to a change in the microstructure for TBCs, such as, the formation and growth of thermally grown oxide (TGO) at interface, phase transformation, porosity decrease in coating [3-5] The TGO formation results in a large compressive stress, which leads to TGO undulation, crack formation, propagation, coalescence and eventually top ceramic coating spallation $[6,7]$. Phase transformation, such as, the metastable $t^{\prime}$ phase undergoes transformations, changing from $t$ ' YSZ into monoclinic $(m)$ YSZ, which results in a volume expansion in TBCs [8]. The porosity decrease in a top ceramic coating reduces the strain tolerance, which is detrimental to the lifetime of thermal cycling [9]. Furthermore, the porosity decrease and pore redistribution can lead to an increase in thermal conductivity, which implies the loss of thermal insulation to the substrate [5]. Therefore, it is essential to develop non-destructive techniques to evaluate the microstructure change in TBCs and TGO growth for predicting the lifetime and failure of TBCs on engine components.

Impedance spectroscopy (IS) is developed as a non-destructive technique which can be used to investigate the TGO growth, phase transformation and degradation of TBCs [10-12]. Wang et al. [13] use the IS testing technique to investigate the TGO growth in TBCs after oxidation at $1100{ }^{\circ} \mathrm{C}$. Anderson et al. [14] study the effects of heat treatment on microstructure, phase composition, and mechanical properties by using IS technique combined with X-ray diffraction, scanning electron microscopy, and microindentation. The IS technique has been applied to several aspects successfully by measuring impedance of a TBC system, such as the degradation of TBCs [15] and failure detection [16]. However, most reports are given about the effect of microstructure features on the impedance of TBCs, whereas less attention is paid on the IS measuring conditions. Based on our previous

\footnotetext{
* Corresponding authors at: Key Laboratory of Key Film Materials \& Application for Equipment (Hunan province), School of Materials Science and Engineering, Xiangtan University, Xiangtan, Hunan 411105, China.

E-mail addresses: lyang@xtu.edu.cn (L. Yang), zhouyc@xtu.edu.cn (Y.C. Zhou).
} 
work [17], it is found that the impedance response is strongly affected by measurement parameters including voltage amplitude, specimen temperature and electrode size. The temperature greatly changes the resistivity of specimen, and results in significant influence on impedance spectra. The influence is ascertained once the relationship between material resistivity and temperature is determined, and thus does not produce measurement error. There is no obvious influence of voltage amplitude on impedance spectra as long as it can ensure that the current is greater than the range of instrument. However, the electrode size brings large error in the measurement error, which also is founded in Ogawa' IS experimental investigation on layer thicknesses of TBCs [18], causing 330\% the maximum measurement error. Therefore, it is necessary to quantify the measurement error for precise quantitative evaluation.

In IS testing technique for TBCs, asymmetric electrode is usually adopted to avoid electric leakage. For example, one is a Pt film electrode with $5 \mathrm{~mm}$ in diameter on ceramic coating, and the other is metal substrate. Consequently, it is reasonable to present the following questions: whether the impedance results are affected by asymmetric electrode, how to affect impedance feature, and how to overcome the errors of measurement results induced by asymmetric electrode. Several experimental investigations find that both electrode geometry and size have the significant effect on impedance feature $[9,19,20]$, and sometimes asymmetric electrode geometry can lead to the spread of an electrical conduction region in TBCs, while it is not reliable to find it in experiments and not to present to measure the divergence of electric field quantitatively. Using finite element method, Deng et al. [21] investigated the effects of TGO growth and conductivity on the IS distribution of TBCs produced by the electron beam physical vapor deposition. The finite element simulation is an effective method to study the influences of asymmetric electrode on IS distribution.

In the present research, a three-dimensional model has been developed to investigate the effects of asymmetric electrode on IS of TBCs. First of all, the distributions of electric field lines, for different frequencies and Pt electrode sizes, are calculated by using the finite element simulation. Then, thickness measurement errors for YSZ and TGO layers due to asymmetric electrode are analyzed quantitatively. Finally, an empirical equation is proposed to modify the thickness measurement errors.

\section{Modeling}

\subsection{The theory foundation}

According to the complex form of Maxwell-Ampere equation, the relationship between magnetic field intensity $(\boldsymbol{H})$, conduction current density $(\boldsymbol{J})$ and electric displacement $(\boldsymbol{D})$ can be represented in the following form:

$\nabla \times \boldsymbol{H}=\boldsymbol{J}+\mathrm{j} \omega \boldsymbol{D}$

where $\omega$ is the angular frequency, and $\mathrm{j}$ is the imaginary unit. Because magnetic field is a no-source field, from Eq. (1) one can obtain

$\nabla \cdot(\nabla \times \boldsymbol{H})=\nabla \cdot(\boldsymbol{J}+\mathrm{j} \omega \boldsymbol{D})=0$

According to the definition of electric displacement $(D)$ in the uniform medium and Ohm's law, the electric displacement $(\boldsymbol{D})$, current density $(\boldsymbol{J})$ and electric field intensity $(\boldsymbol{E})$ are specified, respectively by

$\boldsymbol{D}=\varepsilon_{\mathrm{r}} \varepsilon_{0} \boldsymbol{E}$

$\boldsymbol{J}=\sigma \boldsymbol{E}$

$\boldsymbol{E}=-\nabla V$

where $\varepsilon_{\mathrm{r}}$ and $\varepsilon_{0}$ are the relative permittivity and vacuum permittivity, respectively. $\sigma$ is the conductivity and $V$ is the electric potential. By combining the above equations, the Eq. (2) can be rewritten as [22]

$\nabla \cdot\left(\left(\sigma+\mathrm{j} \omega \varepsilon_{\mathrm{r}} \varepsilon_{0}\right) \nabla V\right)=0$

Then, the Eq. (6) can be deduced as

$\nabla^{2} V=0$

For three-dimensional model depicted in Eq. (7), the control equation is expressed as

$\frac{\partial^{2} V}{\partial x^{2}}+\frac{\partial^{2} V}{\partial y^{2}}+\frac{\partial^{2} V}{\partial z^{2}}=0$

The electrical potential distribution can be obtained by solving the Laplace's equation using the finite element method. In the finite element simulations of IS distribution of TBCs, both conduction currents and displacement currents are used in the frequency domain and the time dependent study types dynamic formulations. And the finite element simulations are carried out by using COMSOL 4.3 software. Based on the Eqs. (3), (4) and (5), as the electrical potential is obtained, the complex current density $(\boldsymbol{J}+\mathrm{j} \omega \boldsymbol{D})$ can be calculated. The complex current $(I)$ can be calculated by integration of the complex current density $(\boldsymbol{J}+\mathrm{j} \omega \boldsymbol{D})$ in the normal direction of the interface under consideration, $I=\int(\boldsymbol{J}+\mathrm{j} \omega \boldsymbol{D}) \cdot d \boldsymbol{n}$, where $\boldsymbol{n}$ represents the normal direction of TBCs interface. Then, the impedance $(Z)$ can be obtained by $Z=U / I=U / \int(\boldsymbol{J}+\mathrm{j} \omega \boldsymbol{D}) \cdot d \boldsymbol{n}$, where $U$ is the applied AC voltage.

\subsection{Geometric and material parameters}

As illustrated in Fig. 1, a specimen with dimensions of $10 \times 10 \mathrm{~mm}^{2}$ is used in the experiment. A platinum (Pt) electrode is prepared on the top ceramic coating surface by ion sputtering (ETD-3000). The typical structure of TBCs used in this paper is consisted of four layers: ceramic (a)

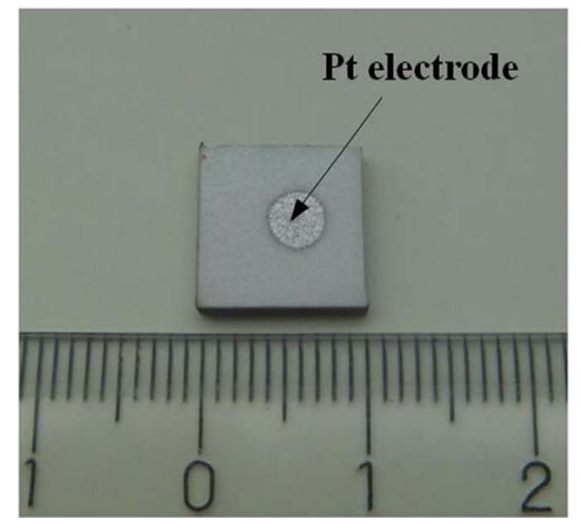

(b)

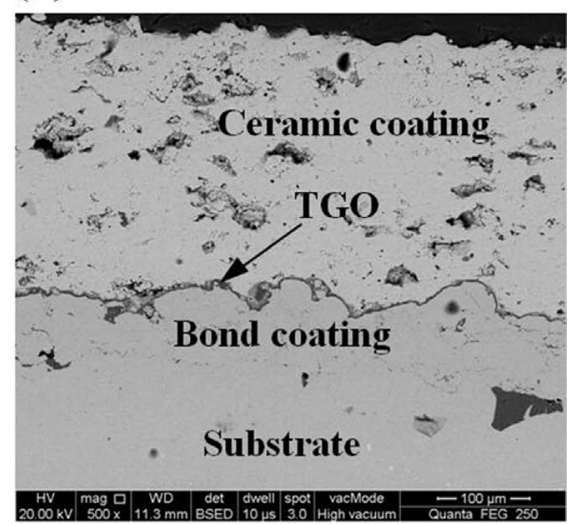

Fig. 1. (a) The sample with dimensions of $10 \times 10 \mathrm{~mm}^{2}$ used in the experiment; (b) the scanning electron micrograph for the cross-section in the APS TBCs. 

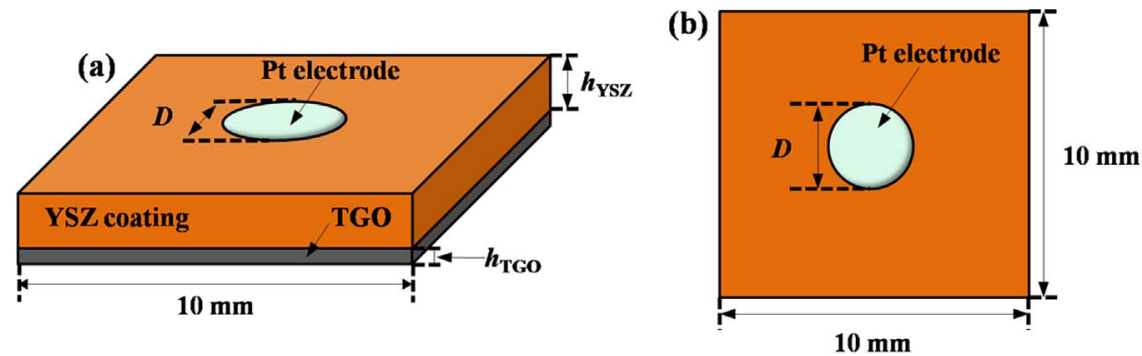

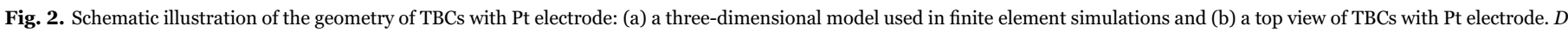
is the diameter of electrode and $L$ is the length of the sample.

coating, bond coating, TGO and substrate (Fig. 1(b)). A three-dimensional model is constructed for the TBCs specimen. Due to the good conductivity of the bond coating and substrate, their influence on the IS distribution can be neglected in the model [21]. Only ceramic coating and TGO layer are included in the model. As shown in Fig. 2, the Pt electrode with diameter of $D$ is attached on the center of the top ceramic coating surface with dimensions of $10 \times 10 \mathrm{~mm}^{2}$, and the bottom of TGO surface acts as the other electrode. In order to study the effect of electrode size, the diameter of the Pt electrode varying from 1 to $9 \mathrm{~mm}$ is discussed. Ceramic coating is a layer with thickness of $h_{\mathrm{YSZ}}$, varying from 150 to $300 \mu \mathrm{m}$, and TGO with thickness of $h_{\mathrm{TGO}}$ changing from 1 to $3 \mu \mathrm{m}$.

Assuming that the YSZ ceramic coating is comprised of continuous uniform grain and the effect of YSZ grain boundary is neglected $[9,23,24]$, the electrical and dielectric properties of various materials at $400{ }^{\circ} \mathrm{C}$ are as follows. The conductivity of YSZ ceramic coating ( $\left.\sigma_{\mathrm{YSZ}}\right)$ can be selected as the one of YSZ grain with the value of $\sigma_{\mathrm{YSZ}}=1 \times 10^{-3} \mathrm{~S} / \mathrm{m}$ [25], the relative permittivity is selected as $\varepsilon_{\mathrm{rYSZ}}=28[21,26]$. For TGO, the influence of the mixed oxide on IS distribution is not considered in the present research. The conductivity and relative permittivity of TGO are selected as $\sigma_{\mathrm{TGO}}=3 \times 10^{-7} \mathrm{~S} / \mathrm{m}$ [27] and $\varepsilon_{\mathrm{rTGO}}=10$ [21], respectively.

\subsection{Boundary conditions}

The three boundary conditions are given as follows: (1) There is no electrical potential drop across the interfaces of Pt/YSZ and YSZ/TGO, the amplitude of applied AC voltage of Pt electrode and the bottom of TGO surface are set as $1 \mathrm{~V}$ and $0 \mathrm{~V}$, respectively; (2) Assuming that no current going out of the TBCs, so the current in the normal direction of the edges between TBCs and outside is set to zero, $(\mathbf{J}+\mathrm{j} \omega \mathbf{D}) \cdot \mathbf{n}=0$; (3) There is no charge accumulation in each element. In other words, the current densities at the interfaces of $\mathrm{Pt} / \mathrm{YSZ}$ and YSZ/TGO are continuous.

\section{Results and discussions}

\subsection{Electric field divergence induced by asymmetric electrode}

Fig. 3 shows the effect of asymmetric electrode geometry on the distribution of electric field line. When the size of Pt electrode is equal to the one of the bottom of TGO surface, it is called symmetric electrode geometry. For symmetric electrode, the electric force lines are parallel to each other, and perpendicular to the electrode plane (see Fig. 3(a), (b)). Compared with the results of symmetric electrode, it is found that the asymmetric electrode geometry induces the spread of an electrical conduction region outside the Pt electrode. The electrical conduction region spreads more widely with the increase of the distance of Pt electrode and YSZ/TGO interface (Fig. 3(c), (d)). This implies the asymmetric electrode increases the electrical conduction region, which results in the measurement errors of IS and affects the precise interpretation of IS distribution of TBCs.

The evolutions of the distribution of electric field line with frequencies are given in Fig. 4. For symmetric electrode, it appears that the distribution of electric field line is independent of frequency, as the frequency varies from low frequency (below $100 \mathrm{~Hz}$ ) to high frequency (above $100 \mathrm{kHz}$ ), the electric force lines are always parallel to each other (see Fig. 4(a)). No divergence of electric field line happens. However, for asymmetric electrode, the distribution of electric field line is significantly dependent on the frequency. In generally, the divergence extent of electric field line becomes large as frequency decreases, which is in agreement with the experimental observation in Tanno et al. [9]. According to the characteristic distribution of electric field lines in different frequencies, the applied voltage frequency can be divided into three stages: low frequency (below $100 \mathrm{~Hz}$ ), medium frequency (in the range of $100 \mathrm{~Hz}$ to100 KHz)

\section{Symmetric electrodes}

(a)

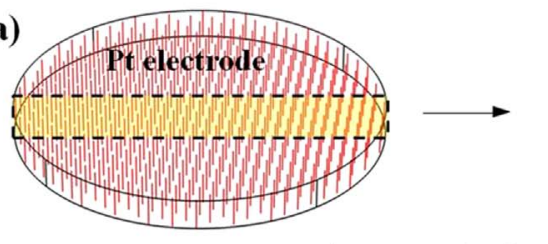

(b)

Pt electrode

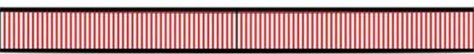

Asymmetric electrodes

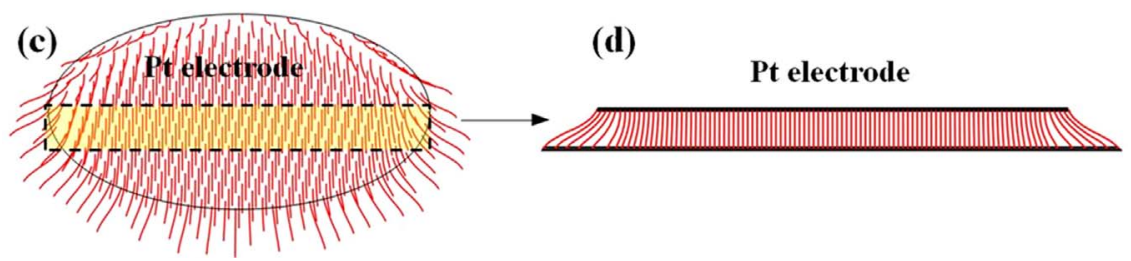

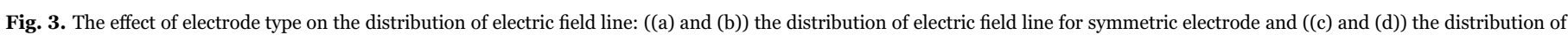
electric field line for asymmetric electrode. 


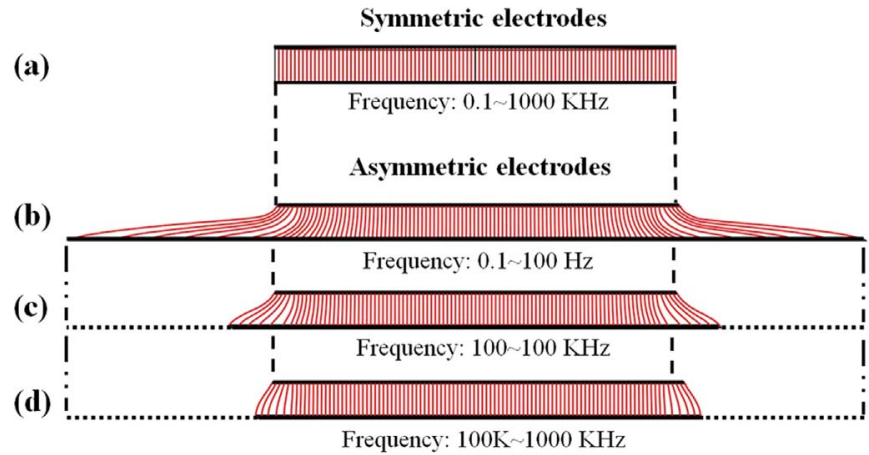

Fig. 4. The variations of the distribution of electric field line with frequencies: (a) symmetric electrode; (b) asymmetric electrode (low frequency); (c) asymmetric electrode (medium frequency) and (d) asymmetric electrode (high frequency).

and high frequency (above $100 \mathrm{kHz}$ ). In the low frequency, the divergence extent of electric field line is the largest in the three frequency ranges, as shown in Fig. 4(b). Moreover, as the frequency increases from 0.1 to $100 \mathrm{~Hz}$, there is no significant change in the distribution of electric field line. The divergence extent of electric field line is obvious in the medium frequency, but the electrical conduction region is much smaller than that in the low frequency (Fig. 4(c)). In addition, the electrical conduction region rapidly decreases as the frequency increases in the range of $100 \mathrm{~Hz}$ to $100 \mathrm{KHz}$. In the high frequency, the divergence extent of electric field line is very small, as shown in Fig. 4(d). Furthermore, the increase of frequency has little effect on the variation of electric field line.

The Nyquist diagram (complex plane plot) and Bode plot of the IS distribution of TBCs with the electrode diameter of $3 \mathrm{~mm}$ are shown in Fig. 5. Two typical semi-circles occur in the range of medium (in the range of $100-100 \mathrm{kHz}$ ) and high frequency (above $100 \mathrm{kHz}$ ), which corresponds to the impedance of TGO and YSZ [13,28], respectively (Fig. 5(a)). It is found that the frequency response of asymmetric electrode is similar to that of symmetric electrode, but the amplitude of the impedance is less than that of symmetric electrode. Moreover, the impedance reduction of TGO is much more than that of YSZ due to the effect of asymmetric electrode. Because the semi-circle for YSZ is in the high frequency, while TGO in the medium frequency. According to Fig. 4, the divergence extent of electric field line in the medium frequency is much larger than that in the high frequency, which induces the spread of an electrical conduction region in TGO. As shown the Bode plot in Fig. 5(b), there are two peaks around $10^{3}-$ $10^{4} \mathrm{~Hz}$ and $10^{7} \mathrm{~Hz}$, which corresponds to the responses of TGO and YSZ. It appears that the effect of asymmetric electrode on Bode plot is

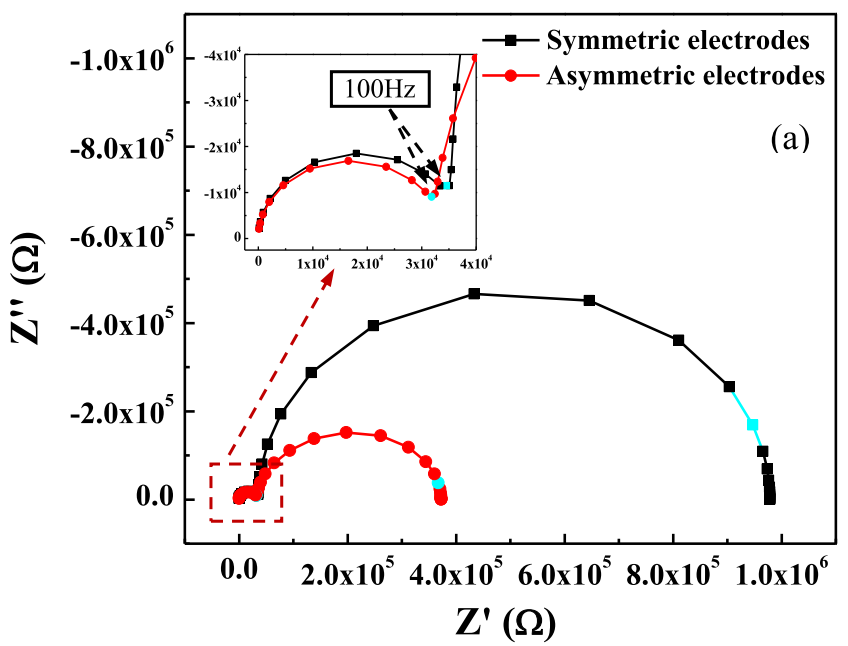

Table 1

The measurement errors of YSZ and TGO thickness for different electrode type.

\begin{tabular}{|c|c|c|c|c|c|c|}
\hline Electrode type & $\begin{array}{l}h_{\mathrm{YsZ}} \text { in } \\
\text { model } \\
(\mu \mathrm{m})\end{array}$ & $\begin{array}{l}h_{\mathrm{YSZ}} \\
\text { from } \\
\text { IS } \\
(\mu \mathrm{m})\end{array}$ & Error $_{\mathrm{YSZ}}$ & $\begin{array}{l}h_{\mathrm{TGO}} \text { in } \\
\text { model } \\
(\mu \mathrm{m})\end{array}$ & $\begin{array}{l}h_{\mathrm{TGO}} \\
\text { from IS } \\
(\mu \mathrm{m})\end{array}$ & Error $_{\mathrm{TGO}}$ \\
\hline $\begin{array}{l}\text { Symmetric } \\
\text { electrode }\end{array}$ & 250 & 250 & $0.00 \%$ & 2.00 & 2.00 & $0.00 \%$ \\
\hline $\begin{array}{l}\text { Asymmetric } \\
\text { electrode }\end{array}$ & 250 & 222 & $10.9 \%$ & 2.00 & 0.725 & $63.8 \%$ \\
\hline
\end{tabular}

mainly focused on the TGO peak of medium frequency, which leads to the decrease of the TGO peak of phase angle. However, the effect of asymmetric electrode on the YSZ peak in the high frequency and electrode response in the low frequency can be neglected, which proves the validity of results in Fig. 4.

The IS analysis is performed using Zview impedance analysis software. The resistances of YSZ and TGO can be obtained by fitting the quasi semicircles in Fig. 5(a), using an equivalent circle of a CPE paralleled with a resistor (R). According to the definition of resistor, the expression of resistor can be represented in the following form:

$R=h /(\sigma A)$

where $h$ and $A$ are the thickness and area of electrical conduction region, respectively. $\sigma$ is the conductivity. When resistance $R$, conductivity $\sigma$ and area $A$ are given, the corresponding thicknesses of both YSZ and TGO layers can be obtained. Due to asymmetric electrode, the electric field line passing through ТВCs looks like a conical shape. This induces the increase of area of electrical conduction region, which leads to the measurement errors of YSZ and TGO thickness, as shown in Table 1. Because of the existence of asymmetric electrode, the measurement errors of YSZ and TGO thickness are $10.9 \%$ and $63.8 \%$, respectively. It appears that the error in TGO thickness measurement is much larger than that in YSZ, this is because the divergence area of electrical conduction region in TGO is much wider.

Fig. 6 shows the divergence extent of electric field line with different size of Pt electrode. It appears that the spread of electrical conduction region is almost independent of the size of Pt electrode. The area of spread of electrical conduction is all the same with the increase of $\mathrm{Pt}$ electrode size. As shown the Nyquist plot in Fig. 7(a), the TGO and YSZ (see the inset) semi-circles become smaller with increase in the size of Pt electrode. The reason is that area of electrical conduction region increases with the size of Pt electrode, which leads to the decrease of TGO and YSZ resistance (see Eq. (9)). In the Bode plot (see Fig. 7(b)), as Pt electrode size increases, the TGO response appears to increase,

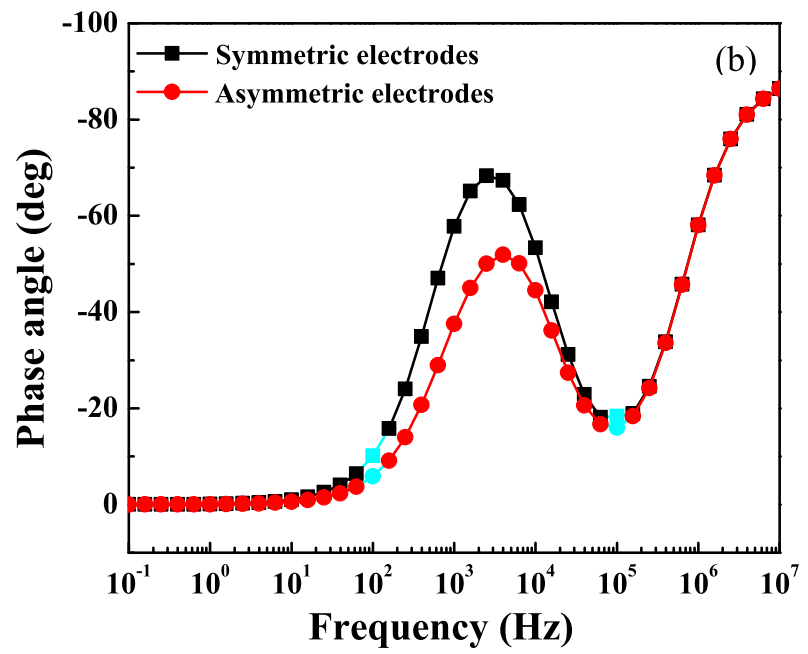

Fig. 5. Typical (a) Nyquist and (b) Bode plots of the IS of TBCs with the electrode diameter of $3 \mathrm{~mm}$ for different electrode type. 


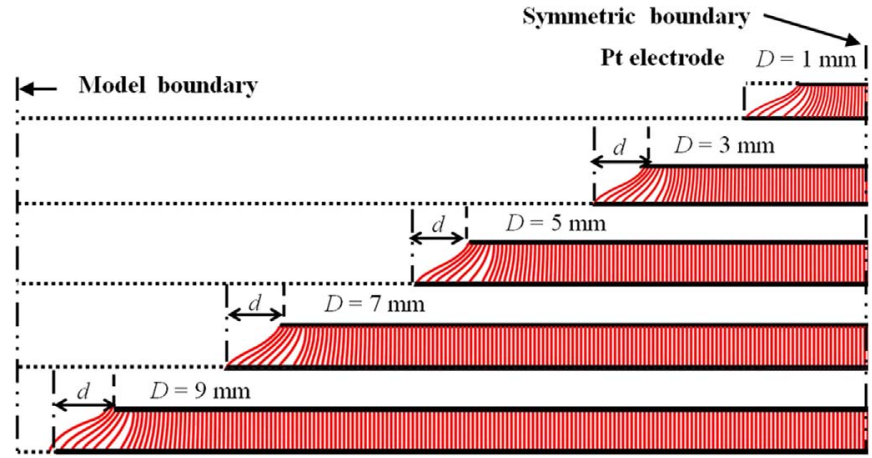

Fig. 6. The influence of electrode size on the distribution of electric field line: (a) $D=1 \mathrm{~mm}$; (b) $D=3 \mathrm{~mm}$; (c) $D=5 \mathrm{~mm}$; (d) $D=7 \mathrm{~mm}$ and (e) $D=9 \mathrm{~mm}$.

whilst YSZ and electrode responses are largely unaffected. The results are in agreement with that obtained by Anderson et al. [19].

The impedance response of TBCs with different Pt electrode sizes is measured by IS testing experimentally [17]. As shown in the Nyquist plot (Fig. 8(a)), the curve obviously shrinks with increasing Pt electrode size. Furthermore, it can be seen in the Bode plot (Fig. 8(b)) that no obvious effect on the YSZ peak can be seen with enlarging Pt electrode size, whereas the TGO peak exhibits an increase as the electrode size increases from 3 to $9 \mathrm{~mm}$. This result is in good agreement with that based on finite element simulations.

\subsection{Error of thickness measurement using asymmetric electrode}

The divergence extent of electric field line is not only dependent on the frequency of applied voltage, but also the thickness of YSZ and TGO. As the diameter of Pt electrode and TGO thickness are selected as $3 \mathrm{~mm}$ and $2 \mu \mathrm{m}$, the distribution of electric field line with different YSZ thickness in the frequency of $10^{4} \mathrm{~Hz}$ is shown in Fig. 9. The spread of electrical conduction region becomes wider with the increase of YSZ thickness. As shown the Nyquist plot in Fig. 10(a), the semi-circle for YSZ response increases with increasing the thickness of YSZ. The reason is that the resistance rises with the YSZ thickness, while the size of YSZ semi-circle is proportional to the YSZ resistance. On the contrary, the semi-circle for TGO response reduces with the increase of the YSZ thickness, which is attributed to the distribution of electric field in TBCs. Due to the spread of electrical conduction region in TGO, the TGO resistance decreases, which results in the reduction of the size of TGO semi-circle. The above results are consistent with the experiment results obtained by Anderson et al. [19]. The Bode plot of the IS of TBCs with different YSZ thickness are shown in Fig. 10(b). The YSZ

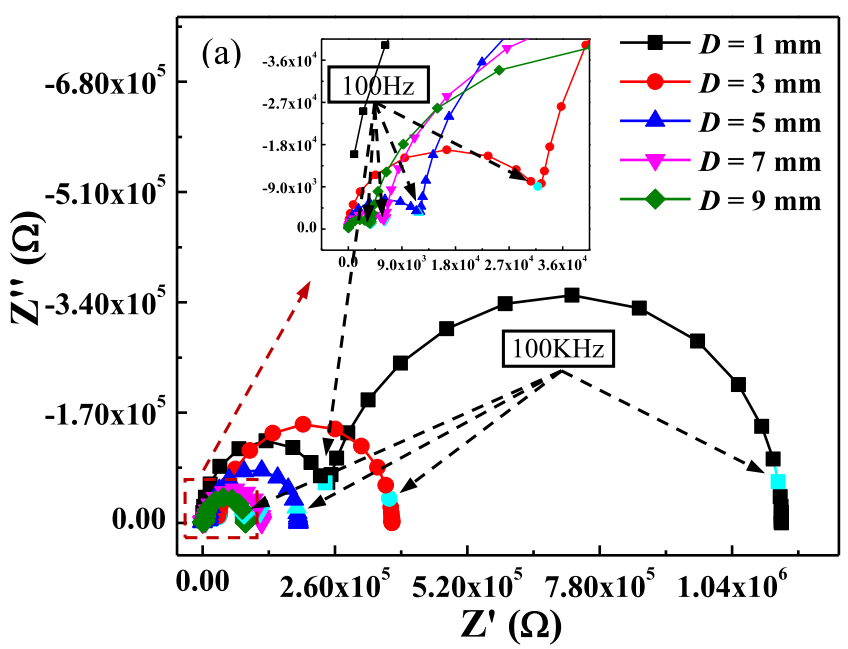

thickness has no influence on the YSZ peak in the high frequency and electrode response in the low frequency, while the TGO peak in the medium frequency reduces with increase of YSZ thickness. The similar phenomenon is observed by Anderson et al. [19]. Table 2 gives the measurement errors of YSZ and TGO thickness due to the effect of YSZ thickness. The thickness measurement error rises with the increase of YSZ thickness. The thickness measurement errors of YSZ and TGO increase from 8.7 to $14.5 \%$ and 54.2 to $69.6 \%$, respectively. The spread of electrical conduction region in TGO is wider than that in YSZ, which results in the larger measurement error of TGO thickness.

The effect of TGO thickness on distribution of electric field line is similar to that of YSZ thickness, as shown in Fig. 11. As the YSZ thickness is selected as $250 \mu \mathrm{m}$, the spread of electrical conduction region becomes wider with TGO thickness varying from 1 to $3 \mu \mathrm{m}$. As shown in Fig. 12(a) for the Nyquist plot, the resistance rises with the TGO thickness, which leads to the increase of the semi-circle for TGO response. The semi-circle for YSZ response is almost independent of TGO thickness. Fig. 12(b) shows the calculated Phase angle vs. Log (freq) plots. The effect of TGO thickness is mainly focused on the TGO peak in the medium frequency. With increase in the TGO thickness, TGO peak rises and shifts to higher frequency. The variation trend is in agreement with the results obtained by Deng et al. [21]. Because of the effect of TGO thickness, the measurement errors of YSZ and TGO thickness are given in Table 3. The trend of measurement errors is similar to that of YSZ thickness. As the TGO thickness varies from 1 to $3 \mu \mathrm{m}$, the measurement errors of YSZ and TGO thickness increase from 9.4 to $12.6 \%$ and 53.7 to $63.9 \%$, respectively. It is found that the error in TGO thickness measurement is much larger than that in YSZ. This also implies the spread of electrical conduction region in TGO.

\subsection{Error modification for thickness measurement using asymmetric electrode}

The measurement errors of YSZ and TGO thickness with different Pt electrode size are shown in Table 4. Both the measurement errors of YSZ and TGO thickness decrease with increasing the size of Pt electrode. As Pt electrode diameter varies from 1 to $9 \mathrm{~mm}$, the measurement errors of YSZ and TGO thickness decrease from 26.2\% to $1.5 \%$ and $89.3 \%$ to $23.6 \%$, respectively. The above results can be attributed to the following reason. As no spread of electrical conduction happens, the real thickness can be obtained by

$h=R \sigma A$

where $A$ is area of electrical conduction region, which can be represented as $A=\pi(D / 2)^{2}, D$ is Pt electrode diameter. Due to asymmetric electrode, the electrical conduction region spreads, and

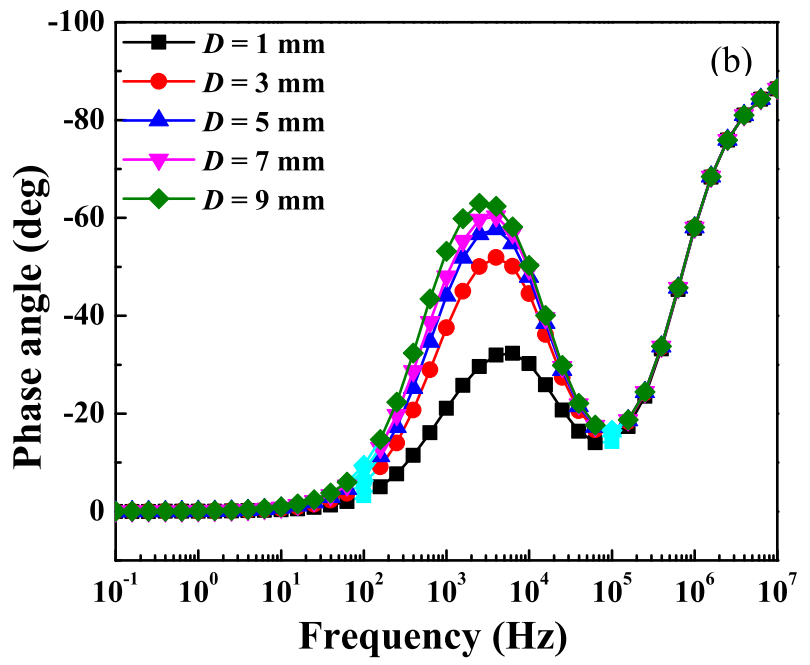

Fig. 7. Typical (a) Nyquist and (b) Bode plots of the IS of TBCs for different electrode size. 

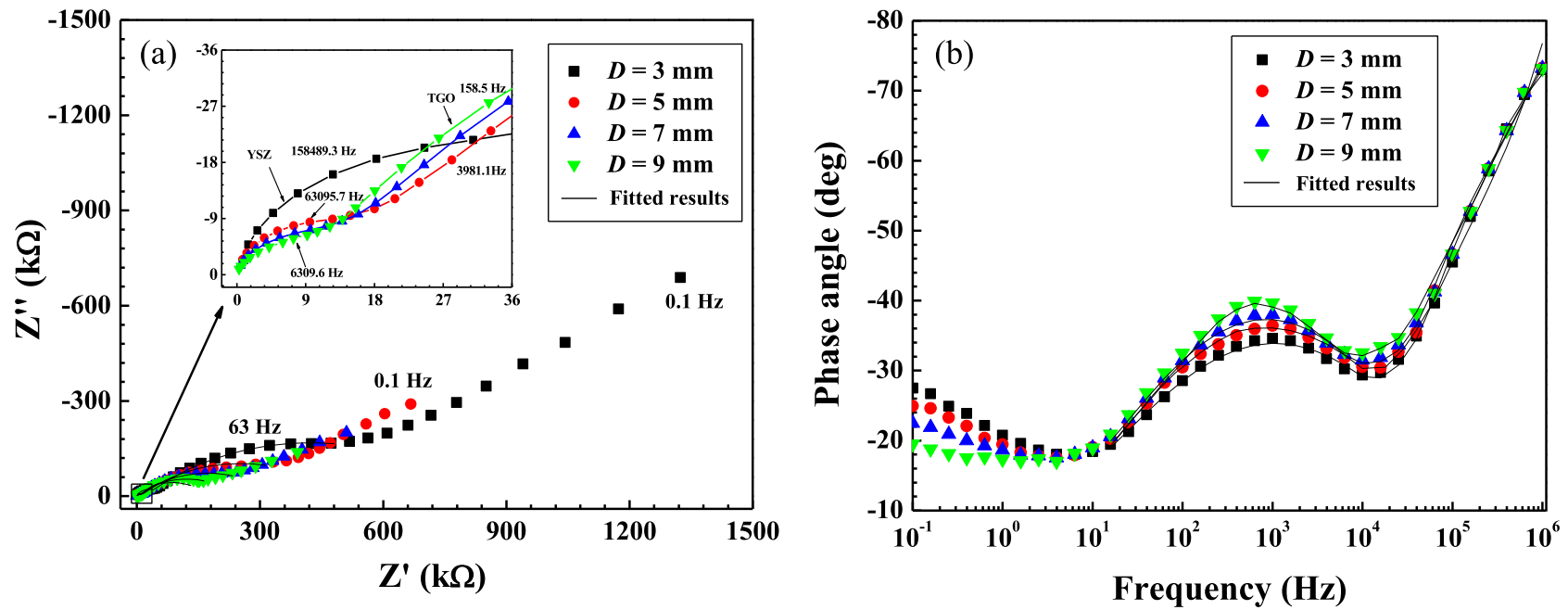

Fig. 8. Measured impedance spectra (IS) of TBCs with different Pt electrode size in (a) Nyquist plot, (b) Bode plot of phase angle vs. frequency [17].

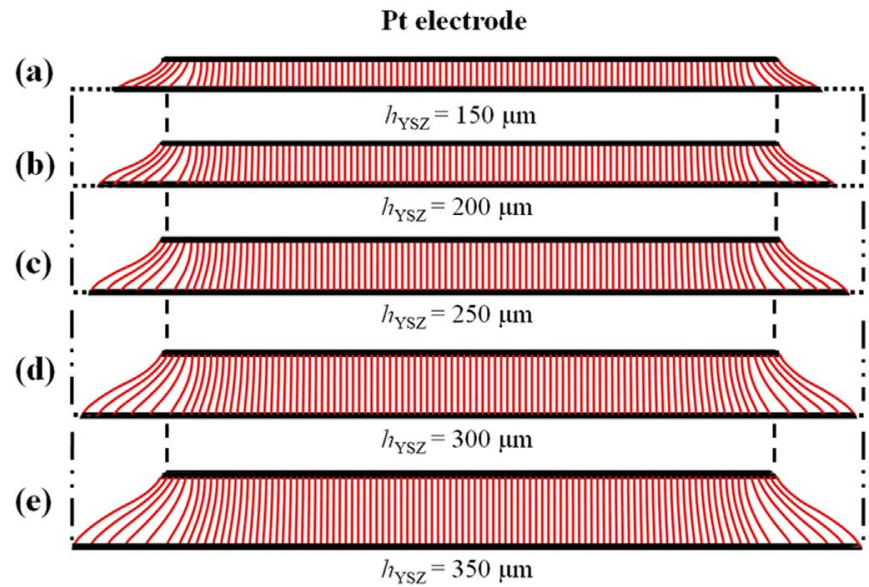

Fig. 9. The influence of YSZ thickness on the distribution of electric field line: (a) $h_{\mathrm{YSZ}}=150 \mu \mathrm{m}$; (b) $h_{\mathrm{YSZ}}=200 \mu \mathrm{m}$; (c) $h_{\mathrm{YSZ}}=250 \mu \mathrm{m}$; (d) $h_{\mathrm{YSZ}}=300 \mu \mathrm{m}$ and (e) $h_{\mathrm{YSZ}}=350 \mu \mathrm{m}$.

the resistances of both YSZ and TGO decrease, the measured thickness can be described as

$h^{*}=R^{*} \sigma A$

where $R^{*}=h /\left(\sigma A^{*}\right), A^{*}$ is defined as $A^{*}=\pi(D / 2+d)^{2}, d$ is the spread

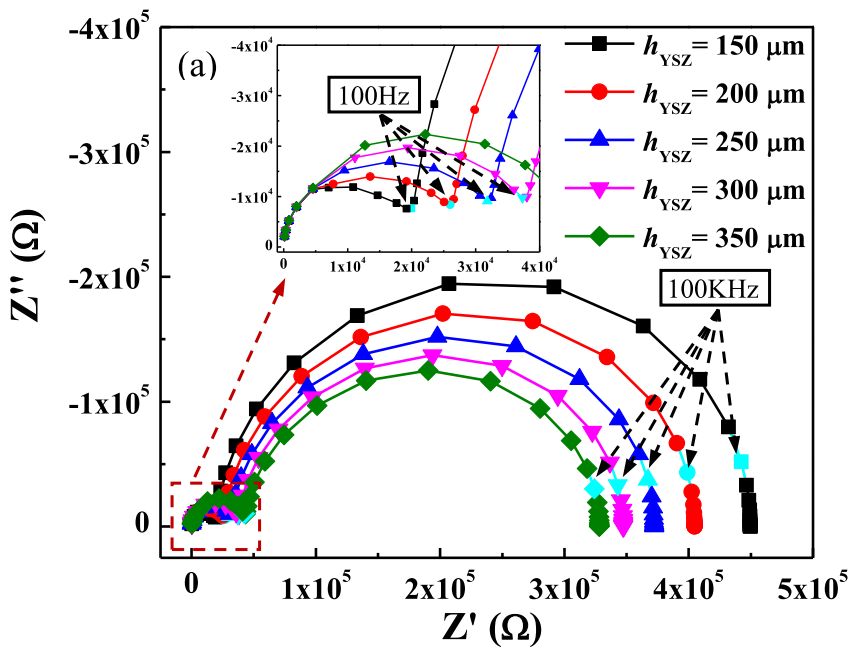

Table 2

The measurement errors of YSZ and TGO thickness for different YSZ thickness.

\begin{tabular}{llllll}
\hline $\begin{array}{l}h_{\mathrm{YSZ}} \text { in model } \\
(\mu \mathrm{m})\end{array}$ & $\begin{array}{l}h_{\mathrm{YSZ}} \text { from } \\
\text { IS }(\mu \mathrm{m})\end{array}$ & Error & $\begin{array}{l}h_{\mathrm{TGO}} \text { in } \\
\text { model }(\mu \mathrm{m})\end{array}$ & $\begin{array}{l}h_{\mathrm{TGO}} \text { from } \\
\text { IS }(\mu \mathrm{m})\end{array}$ & Error $_{\mathrm{TGO}}$ \\
\hline 150 & 137 & $8.65 \%$ & 2 & 0.916 & $54.20 \%$ \\
200 & 182 & $9.21 \%$ & 2 & 0.806 & $59.70 \%$ \\
250 & 222 & $10.90 \%$ & 2 & 0.725 & $63.80 \%$ \\
300 & 261 & $12.80 \%$ & 2 & 0.661 & $66.90 \%$ \\
350 & 299 & $14.50 \%$ & 2 & 0.608 & $69.60 \%$ \\
\hline
\end{tabular}

length of electrical conduction region (see Fig. 10). According to the definition of relative error, the relative error of thickness is specified by

$\Delta=\frac{h-h^{*}}{h}=\frac{4}{\frac{D^{2}}{D d+d^{2}}+4}$

Based on the Eq. (12), as the Pt electrode diameter (D) increases, the spread length of electrical conduction is all the same, the measurement error decreases. It is worth noting that the spread length of electrical conduction in TGO is larger than that in YSZ, which is the reason why the larger error is in TGO thickness measurement.

Since the asymmetric electrode geometry is mostly used in the IS testing, the thickness measurement error is inevitable due to the electric field divergence, it is necessary to present a scheme to eliminate the effect of electric field divergence on thickness measurement error.

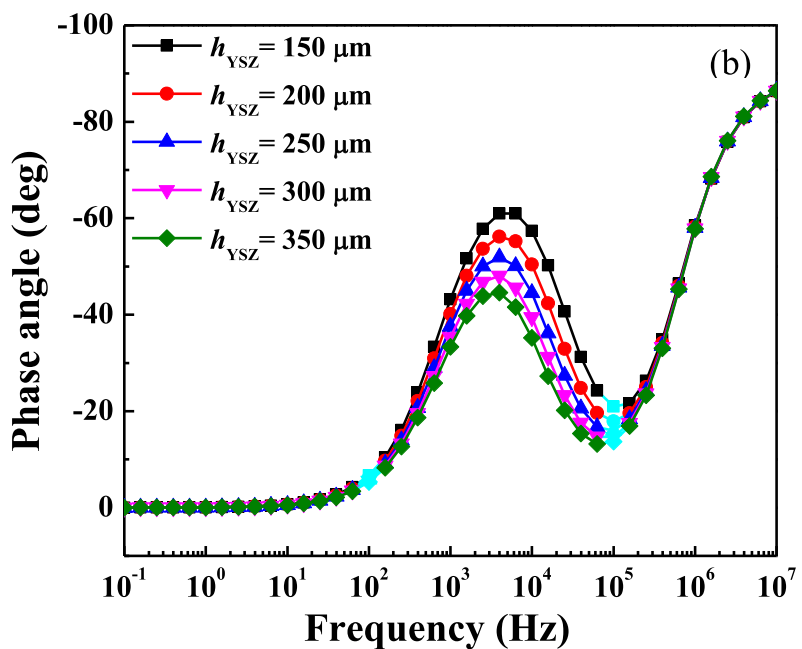

Fig. 10. Typical (a) Nyquist and (b) Bode plots of the IS of TBCs for different YSZ thickness. 


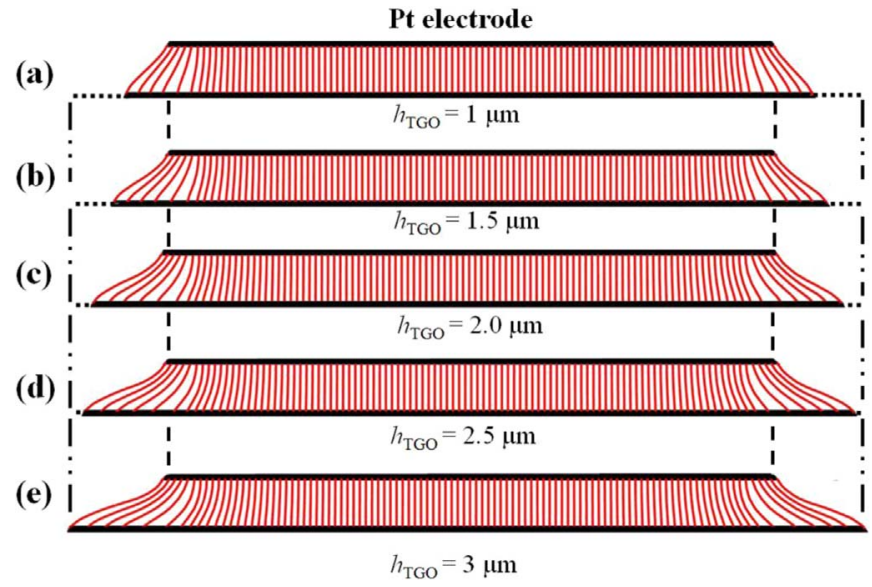

Fig. 11. The influence of TGO thickness on the distribution of electric field line: (a) $h_{\mathrm{TGO}}=1 \mu \mathrm{m}$; (b) $h_{\mathrm{TGO}}=1.5 \mu \mathrm{m}$; (c) $h_{\mathrm{TGO}}=2 \mu \mathrm{m}$; (d) $h_{\mathrm{TGO}}=2.5 \mu \mathrm{m}$ and (e) $h_{\mathrm{TGO}}=3 \mu \mathrm{m}$.

Based on results in Table 4, the relationship between the thickness measurement errors and electrode size can be obtained by fitting the result (see Fig. 13)

$\Delta_{\text {hYSZ }}=-0.089+\frac{0.111}{\sqrt{D / L}}$

$\Delta_{h_{T G O}}=1.21-1.026 \sqrt{D / L}$

Then the modified measurement thickness of YSZ and TGO $\left(h^{* *}\right)$ can be obtained as

$h_{\mathrm{YSZ}}^{* *}=\frac{h_{\mathrm{YSZ}}^{*}}{1-\Delta_{h \mathrm{YSZ}}}=\frac{h_{\mathrm{YSZ}}^{*}}{1-\left(-0.089+\frac{0.111}{\sqrt{D / L}}\right)}$

$h_{\mathrm{TGO}}^{* *}=\frac{h_{\mathrm{TGO}}^{*}}{1-\Delta_{h \mathrm{TGO}}}=\frac{h_{\mathrm{TGO}}^{*}}{1-(1.21-1.026 \sqrt{D / L})}$

\section{Conclusions}

The effects of asymmetric electrode on the IS testing of TBCs have been studied by solving the three-dimensional electric field equations numerically. The distribution of electric field is intensively investigated at different electrode size, voltage frequency, YSZ and TGO thickness. The errors and their modification in thickness measurement are also analyzed. The main conclusions include:

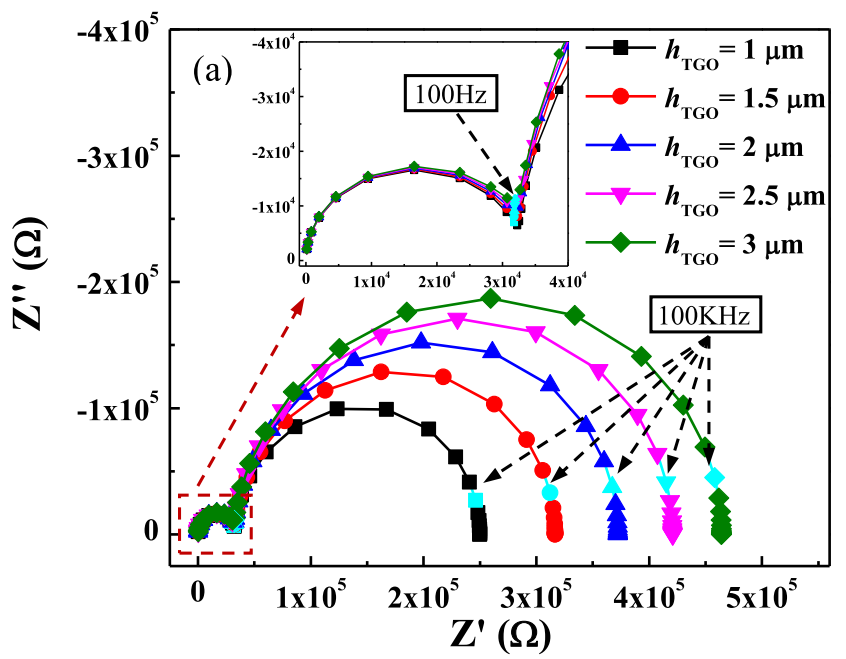

Table 3

The measurement errors of YSZ and TGO thickness for different TGO thickness.

\begin{tabular}{llllll}
\hline $\begin{array}{l}h_{\mathrm{TGO}} \text { in model } \\
(\mu \mathrm{m})\end{array}$ & $\begin{array}{l}h_{\mathrm{TGO}} \text { from } \\
\text { IS }(\mu \mathrm{m})\end{array}$ & Error $_{\mathrm{TGO}}$ & $\begin{array}{l}h_{\mathrm{YSZ}} \text { in model } \\
(\mu \mathrm{m})\end{array}$ & $\begin{array}{l}h_{\mathrm{YSZ}} \text { from } \\
\text { IS }(\mu \mathrm{m})\end{array}$ & Error $_{\mathrm{YSZ}}$ \\
\hline 1 & 0.463 & $53.70 \%$ & 250 & 226 & $9.35 \%$ \\
1.5 & 0.605 & $59.70 \%$ & 250 & 224 & $10.10 \%$ \\
2 & 0.725 & $63.80 \%$ & 250 & 222 & $10.90 \%$ \\
2.5 & 0.828 & $66.90 \%$ & 250 & 221 & $11.70 \%$ \\
3 & 0.922 & $69.30 \%$ & 250 & 218 & $12.60 \%$ \\
\hline
\end{tabular}

Table 4

The measurement errors of YSZ and TGO thickness for different electrode size.

\begin{tabular}{lllllll}
\hline $\begin{array}{l}D \\
(\mathrm{~mm})\end{array}$ & $\begin{array}{l}h_{\mathrm{YSZ}} \text { in } \\
\text { model } \\
(\mu \mathrm{m})\end{array}$ & $\begin{array}{l}h_{\mathrm{YSZ}} \text { from } \\
\text { IS }(\mu \mathrm{m})\end{array}$ & Error $\mathrm{YSZ}$ & $\begin{array}{l}h_{\mathrm{TGO}} \text { in } \\
\text { model } \\
(\mu \mathrm{m})\end{array}$ & $\begin{array}{l}h_{\mathrm{TGO}} \\
\text { from IS } \\
(\mu \mathrm{m})\end{array}$ & Error $_{\mathrm{TGO}}$ \\
\hline 1 & 250 & 184 & $26.20 \%$ & 2 & 0.214 & $89.30 \%$ \\
3 & 250 & 222 & $10.90 \%$ & 2 & 0.725 & $63.80 \%$ \\
5 & 250 & 232 & $7.15 \%$ & 2 & 1.04 & $47.80 \%$ \\
7 & 250 & 236 & $5.73 \%$ & 2 & 1.27 & $36.30 \%$ \\
9 & 250 & 246 & $1.52 \%$ & 2 & 1.53 & $23.60 \%$ \\
\hline
\end{tabular}

(1) Electric field divergence in TBCs measurement induced by asymmetric electrode is inevitable. The distribution of electric field line is significantly dependent on the applied voltage frequency. The divergence extent of electric field line becomes large as frequency decreases, which results in the measurement errors of IS and affects the precise interpretation of IS of TBCs.

(2) The spread of electrical conduction region becomes wider with the increase of YSZ and TGO thickness, which results in the increase of the thickness measurement error. As the YSZ thickness varies from 150 to $350 \mu \mathrm{m}$, the measurement errors of YSZ and TGO increase from $8.7 \%$ to $14.5 \%$ and $54.2 \%$ to $69.6 \%$, respectively. The trend of measurement error is similar to that of YSZ thickness. As the TGO thickness varies from 1 to $3 \mu \mathrm{m}$, the measurement errors of YSZ and TGO increase from $9.4 \%$ to $12.6 \%$ and $53.7 \%$ to $63.9 \%$, respectively. It appears that the error in TGO is much larger than that in YSZ.

(3) A scheme to eliminate the effect of electric field divergence on thickness measurement error has been presented, which quantifies the relationship between the measurement errors and electrode size.

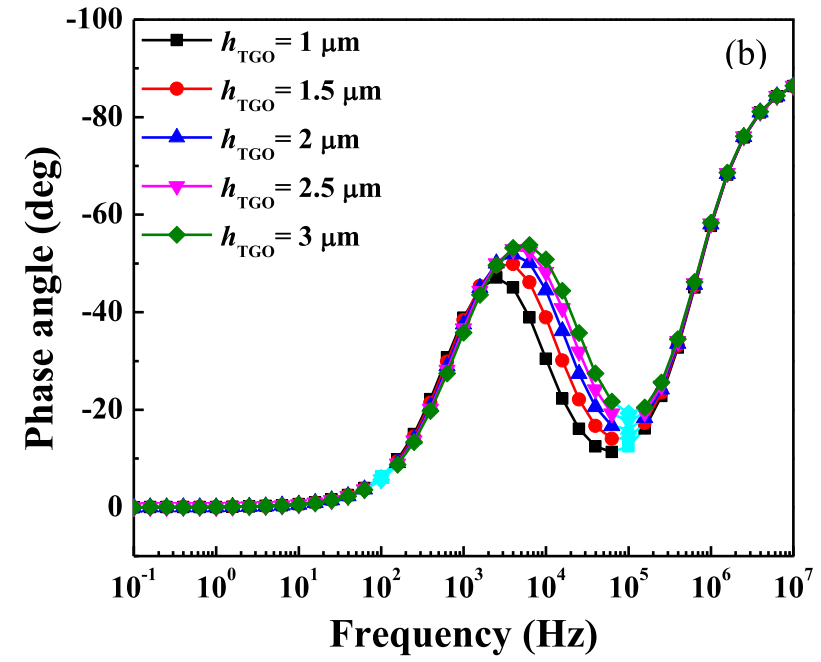

Fig. 12. Typical (a) Nyquist and (b) Bode plots of the IS of TBCs for different TGO thickness. 

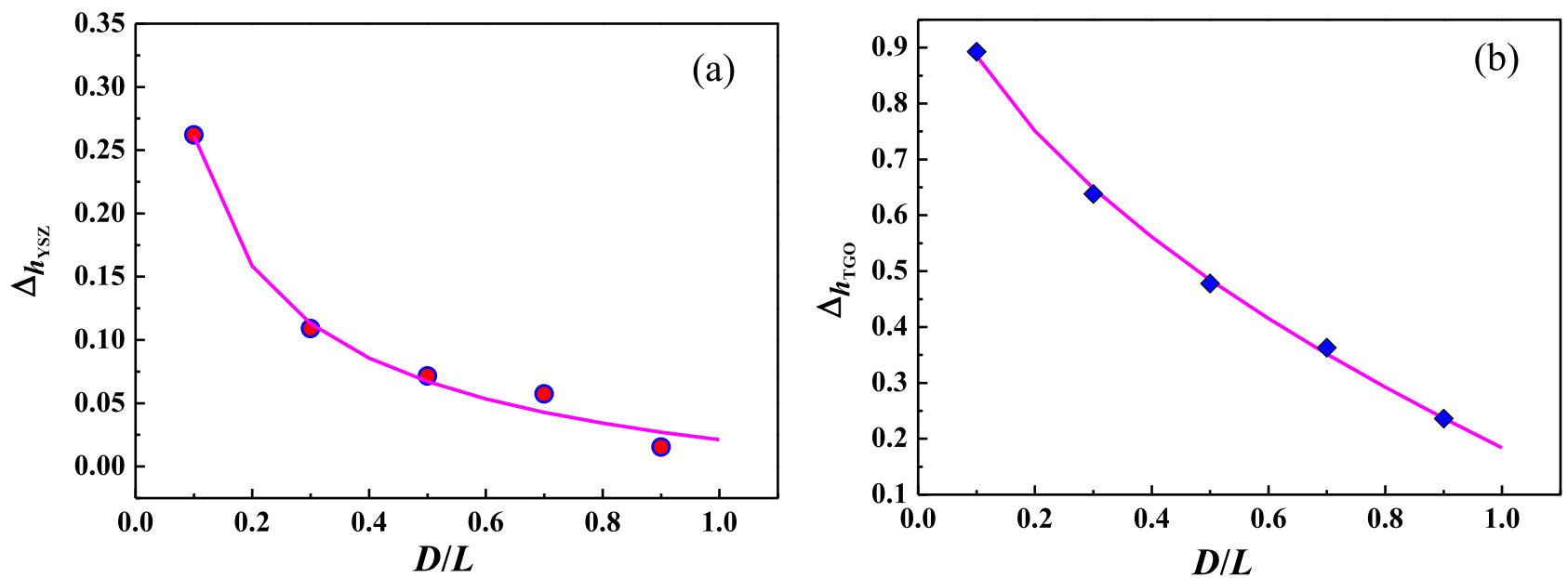

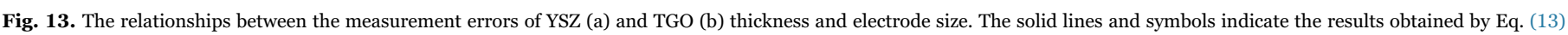
and finite element simulations, respectively.

\section{Acknowledgments}

This work was supported by the National Natural Science Foundation of China (Grant nos. 51590891, 11272275, 11472237, 11602211 and 11432014), the Distinguished Young Scholar Foundation of Hunan Province (Grant No. 2016JJ1014) and the Outstanding Youth Foundation of Hunan Provincial Education Department (Grant No. 16B249).

\section{References}

[1] N.P. Padture, M. Gell, E.H. Jordan, Thermal barrier coatings for gas-turbine engine applications, Science 296 (2002) 280-284.

[2] D.R. Clarke, M. Oechsner, N.P. Padture, Thermal barrier coatings for more efficient gas-turbine engines, MRS Bull. 37 (2012) 891-898.

[3] H.B. Zhao, M.R. Begley, A. Heuer, R.S. Moshtaghin, H.N.G. Wadley, Reaction, transformation and delamination of samarium zirconate thermal barrier coatings, Surf. Coat. Technol. 205 (2011) 4355-4365.

[4] J. Wu, H.B. Guo, M. Abbas, S.K. Gong, Evaluation of plasma sprayed YSZ thermal barrier coatings with the CMAS deposits infiltration using impedance spectroscopy, Prog. Nat. Sci. -Mater. 22 (2012) 40-47.

[5] J. Zhang, V. Desai, Determining thermal conductivity of plasma sprayed TBC by electrochemical impedance spectroscopy, Surf. Coat. Technol. 190 (2005) 90-97.

[6] B.W. Lv, H. Xie, R. Xu, X.L. Fan, W.X. Zhang, T.J. Wang, Effects of sintering and mixed oxide growth on the interface cracking of air-plasma-sprayed thermal barrier coating system at high temperature, Appl. Surf. Sci. 360 (2016) 461-469.

[7] W. Zhu, L. Yang, J.W. Guo, Y.C. Zhou, C. Lu, Determination of interfacial adhesion energies of thermal barrier coatings by compression test combined with a cohesive zone finite element model, Int. J. Plast. 64 (2015) 76-87.

[8] A. Loganathan, A.S. Gandhi, Effect of phase transformations on the fracture toughness of $t^{\prime}$ yttria stabilized zirconia, Mater. Sci. Eng. A 556 (2012) 927-935.

[9] M. Tanno, K. Ogawa, T. Shoji, Influence of asymmetric electrode geometry on an impedance spectrum of a plasma-sprayed thermal barrier coating system, Surf. Coat. Technol. 204 (2010) 2504-2509.

[10] C. Zhu, A. Javed, P. Li, F. Yang, G.Y. Liang, P. Xiao, A study of the microstructure and oxidation behavior of alumina/yttria-stabilized zirconia $\left(\mathrm{Al}_{2} \mathrm{O}_{3} / \mathrm{YSZ}\right)$ thermal barrier coatings, Surf. Coat. Technol. 212 (2012) 214-222.

[11] A. Shinmi, X. Zhao, P. Xiao, Mechanical and electrical characterization of a phase transformation in thermal barrier coatings, Surf. Coat. Technol. 205 (2010) $2678-2686$
[12] J.W. Byeon, B. Jayaraj, S. Vishweswaraiah, S. Rhee, V.H. Desai, Y.H. Sohn, Nondestructive evaluation of degradation in multi-layered thermal barrier coatings by electrochemical impedance spectroscopy, Mater. Sci. Eng. A 407 (2005) 213-225.

[13] X. Wang, J.F. Mei, P. Xiao, Non-destructive evaluation of thermal barrier coatings using impedance spectroscopy, J. Eur. Ceram. Soc. 21 (2001) 855-859.

[14] P.S. Anderson, X. Wang, P. Xiao, Effect of isothermal heat treatment on plasmasprayed yttria-stabilized zirconia studied by impedance spectroscopy, J. Am. Ceram. Soc. 88 (2005) 324-330.

[15] M.S. Ali, S.H. Song, P. Xiao, Evaluation of degradation of thermal barrier coatings using impedance spectroscopy, J. Eur. Ceram. Soc. 22 (2002) 101-107.

[16] N.Q. Wu, K. Ogawa, M. Chyua, S.X. Mao, Failure detection of thermal barrier coatings using impedance spectroscopy, Thin Solid Films 457 (2004) 301-306.

[17] N.G. Wang, C.F. Li, L. Yang, Y.C. Zhou, W. Zhu, C.Y. Cai, Experimental testing and FEM calculation of impedance spectra of thermal barrier coatings: effect of measuring conditions, Corros. Sci. 107 (2016) 155-171.

[18] K. Ogawa, D. Minkov, T. Shoji, M. Sato, H. Hashimoto, NDE of degradation of thermal barrier coating by means of impedance spectroscopy, NDT E Int. 32 (1999) $177-185$.

[19] P.S. Anderson, X. Wang, P. Xiao, Impedance spectroscopy study of plasma sprayed and EB-PVD thermal barrier coatings, Surf. Coat. Technol. 185 (2004) 106-119.

[20] R. Heung, X. Wang, P. Xiao, Characterisation of PSZ $/ \mathrm{Al}_{2} \mathrm{O}_{3}$ composite coatings using electrochemical impedance spectroscopy, Electrochim. Acta 51 (2006) 1789-1796.

[21] L.F. Deng, Y.S. Xiong, P. Xiao, Modelling and experimental study of impedance spectra of electron beam physical vapour deposition thermal barrier coatings, Surf Coat. Technol. 201 (2007) 7755-7763.

[22] COMSOL Multiphysics 4.3 User's Manual.

[23] J.H. Joo, G.M. Choi, Electrical conductivity of YSZ film grown by pulsed laser deposition, Solid State Ion. 177 (2006) 1053-1057.

[24] W. Zhao, I.J. Kim, J. Gong, Influence of thickness on the electrical conductivity of YSZ electrolytes, J. Ceram. Soc. Jpn. 118 (2010) 550-554.

[25] F.E.G. Henn, R.M. Buchanan, N. Jiang, D.A. Stevenson, Permittivity and AC conductivity in yttria-stabilized zirconia, Appl. Phys. A 60 (1995) 515-519.

[26] J. Fleig, S. Rodewald, J. Maier, Microcontact impedance measurements of individual highly resistive grain boundaries: general aspects and application to acceptor-doped $\mathrm{SrTiO}_{3}$, J. Appl. Phys. 87 (2000) 2372-2381.

[27] F. Yang, A. Shinmi, P. Xiao, Electrical and dielectric properties of thermally grown oxide (TGO) on fecralloy substrate studied by impedance spectroscopy, Adv. Ceram. Coat. Interfaces IV (2010) 87-95.

[28] X. Wang, J. Mei, P. Xiao, Determining oxide growth in thermal barrier coatings (TBCs) non-destructively using impedance spectroscopy, J. Mater. Sci. Lett. 20 (2001) 47-49. 